TITLE PAGE: Gender gap in self-rated health: A cohort perspective

Corresponding author: Marta Gil-Lacruz

Department of Psychology and Sociology. Health Science Faculty. University of

Zaragoza

Domingo Miral s/n Zaragoza, 50009 (Spain)

Telephone: (+34) 976761000 ext. 4443

Email: $\underline{\text { mglacruz@ unizar.es }}$

ORCID: 0000-0001-8261-058X

Ana Isabel Gil-Lacruz

Department of Management. University of Zaragoza. School of Engineering and Architecture

C. María de Luna, 3. Edificio Betancourt, Campus Río Ebro Zaragoza, 50018 (Spain)

Telephone: (+34) 976761000 ext. 5206

Email: anagil@unizar.es

ORCID: 0000-0002-7994-923X

Isabel Aguilar Palacio

Department of Microbiology, Preventive Medicine and Public Health

Medicine Faculty. University of Zaragoza C/ Domingo Miral, s/n Zaragoza, 50009

(Spain)

Telephone: (+34) 976761757

Email: iaguilar@unizar.es

ORCID: 0000-0001-7293-701X 


\section{Gender gap in self-rated health: A cohort perspective}

\section{ABSTRACT}

Background: The relation among gender differences and culture in self-rated health is not only bidirectional and static. In this sense, it is important to difference between effects of age and cohort. The aim of this study is analyzing gender gap differences in self-rated health from a generational perspective in order to explore health diversity from a multidisciplinary approach with policy implications.

Methods: We used data drawn from the European Health Interview Survey for 8 Eastern European Countries and EUROSTAT from 2006 through 2009. We conducted multilevel analyses to understand individual and national health determinants of self-rated health by gender and if national differences remain after controlling from micro variables. In order to analyse the role of equity (Gini Quartile) and capacity to create richness (GDPpc Quartile) in gender differences, Oaxaca analyses were developed.

Results: Self-rated health gender gap increases with age. Individual characteristics, such as educational level or smoking influence the citizens' perceived health, with a stronger effect on women than on men. Both characteristics (endowment effects) and effects of individual characteristics (coefficient effects) on health are important to understand gender gaps among people from silent generation.

Conclusions: Our research points out that random effects are greater for men than for women. Besides, random effects might be explained to certain extent by both indexes (Gini and GDPpc). The combined effects of gender, cohort and geographical differences on self-rated health have to be taken into account to figure out public health policies.

Keywords: health gender gap; self-rated health; wealth; economic inequity; health international comparison; European Health Interview Survey; health cohorts research. 


\section{Gender gap in self-rated health: A cohort perspective}

\section{Background}

Women in Europe live on average 10 years in ill health [1-3]. Differences in life expectancy between Western and Eastern European countries remains significant [4] and low-educated groups show disadvantages in relation to highly-educated groups [5]. These inequalities are not getting reduced but they are increasing [6]. An international research across 17 European countries found that although this perception changes widely among nations, women has consistently poorest self-reported health than men, even in healthy countries [7].

Self-assessed health is an important indicator of people's health status. It is a comprehensive assessment of the individual's physical, mental and social well-being [8]. A negative perception of this condition is related to worse indicators of acute and chronic morbidity [9], a greater degree of disability, a more frequent use of health resources [10], deficiencies and/or difficulties in the accomplishment of the daily work, domestic and recreational activities [11]. Despite the subjectivity of this measure, the behaviours to which it leads are observable from clinical practice. In this way, self-reported health is a good starting point to study citizens' health, as it is widely regarded as a good predictor of both health care use and mortality [12-13]. As an alternative hypothesis, we wonder if perceptions of health influence physical functioning, for example through the motivation to engage in physical exercise or in the opposite way: through a lack of confidence and interest in completing functional tasks [14]. In order to analysis this casual paths, longitudinal cross-national data could be very useful [14]. In fact, the use of this indicator in health surveys is widely agreed in an international basis [15]. 
Cultural beliefs, attitudes and values play an important role in the self-perception of health [16]. So, different cultures vary in the degree of openness to the public manifestation of symptoms and ailments, being this one factor usually discriminating between men and women [17]. In this sense, gender is a relevant variable associated with culture. For example, Latin culture is more permissive in expressing a negative perception of health by women, but the direction of these results may not have a unidirectional interpretation $[11,18]$.

The relation among gender differences and culture in self-rated health is not only bidirectional and static. In this sense, it is important to difference between effects of age and cohort [19]. Age effects are related to individual aging process. The effects of birth year and consequently the effects of sharing life events and socioeconomic context for a group is measured by cohort effects [20].

For example, the research conducted by Etherington (2017), using longitudinal data from the Panel Study of Income Dynamics, found that, prior to analysing cohorts' effects on self-rated health, women reported worse health than men did. But introducing cohort variables, no gender difference was found with the exception of the oldest population (born from 1924 to 1933) [21].

Other social variables such as socioeconomic status or educational level also influence the self-interpretation of health [14, 22-24]. The influence of these variables tends to occur in an interdependent way [25]. Socioeconomic status can influence health outcomes for example, through access to medical care, and in this way predict the selfassessed health [14]. Research on income inequality provides empirical evidence about the existence of a social gradient in health [26]. On average, people in a low socioeconomic status have worse health than people who are in wealth position. In this way, unequal societies tend to have unequal health [27]. 
Differential exposure model explains gender inequalities in health as the result of a stratification process that assigns different socioeconomic opportunities to men and women [28]. In order to promote a better self-rated health, researcher focus on the different impact of socioeconomic variables by gender. Education attainments benefit more to women than to men in terms of self-rated health [29]. A major presence of women in the labour market contributes also to a better female self-evaluation [30].

Also, it has been argued that disadvantaged groups will fail to perceive and report the presence of illness or health deficits, which may result in misleading assessments of population health, what is called "reporting heterogeneity". From one hand, advantaged populations tend to report higher levels of poor health than disadvantaged populations $[31,32]$. On the other, self-reported questions may have led to underestimation of unhealthy lifestyle such as smoking, overweight, and low physical activity, because people tend to report better lifestyles than they have [33].

Concerning this diversity of perceptions Eckersley (2005: 59-76) [34] questions: "Were the inequity differences affect health fundamentally by material-resulting from differences in material living conditions and experiences? Or were the inequity differences effect of psychosocial-stemming from people 's position in the social hierarchy and their perception of relative disadvantage? To answer to these questions, we have to add social capital influence. According to Biggs, King, Basu and Stuckler (2010) [35] this interpretation emphasizes the income inequality's role in promoting a social net which protect to poor health and illness.

Recently, methodological improvements show that assuming homogeneity in the relationship between the need and use of care tends to underestimate pro-rich inequity [36]. Although self-rated health is frequently used as health indicator, there is little data on its comparability across cultures. Longitudinal data from Finland and Italy showed 
that self-reported health is a useful summary of physical health, but it may predict mortality better in men than in women and be sensitive to cultural environment. Therefore, further research is request about direct gender and cultural combined effect on self-assessed health [37].

The analysis of health inequity also needs to deep on aggregate data at the national level perspective. In this sense, the scientific literature has focused on the influence of the country's wealth (measured by the GDP) and the inequality index of the distribution of the resources (GINI coefficient) [38]. It seems clear that, as wealthier a country is, more resources are available to spend on health promoting social programs and higher incomes have the citizens to invest on health [35]. In the European context, and with data from the European Social Survey (2002, 2004 and 2006), Huijts, Monden and Kraaykamp (2010) [39] showed that countries' GDP per capita is positively associated with self-rated health.

However, this simple interaction depends on the starting point of domestic wealth. In this sense, Deaton (2003) [40] studied how an additional dollar on public health investment has a stronger effect on poor countries than on richer ones. One reason could be that economic growth allows more people going out of poverty in economic unprivileged countries [41]. In fact, income inequality could be a key variable to explain these results. The Wilkinson and Pickett (2006) [42] literature review showed that over $70 \%$ of 168 studies, reported worse health in countries with big income inequality. In spite of this trend, this relationship is not so evident in the European context than in less egalitarian countries as United States [43].

Lastly, differences not only between populations, but also between cohorts, may exist [44-46]. The trend of poor self-reported health in younger birth cohorts is deeply worrying for the affected individuals, and may also have a negative impact on the health care system. For example, we observe that in spite of increased education, higher income and 
lower smoking rates among Baby Boomers in relation to people from Silent Generation, these improvements could be counterbalanced by the adverse effect of increasing body mass index [47].

Interventions in this area should focus on younger adults, especially females. Further research is needed to determine, for example, poor working conditions among young people figure out their state of health [44, 48-50].

\section{Methods}

Data has been drawn from the European Health Interview Survey (EHIS) first wave (EHIS 83/2014). We have focused on Eastern European countries to control for similarities (welfare state and capacity to create richness), in order to understand how differences (equity) lead to different health status. The use of standard questionnaires and guidelines in all countries guarantee comparability [51].

The final sample size was 79,487 individuals living in 8 Eastern European countries (Bulgaria, Check Republic, Hungary, Latvia, Poland, Romania, Slovenia and Slovakia). Sampling units used in Slovenia, Check Republic, Hungary and Latvia were individuals. Bulgaria used households, and Romania, Poland and Slovakiae used dwellings. Estonia was not included due to the lack of information in some of the key variables for this research.

In this study we have considered people from 15 years old. We have repeated estimations by gender and generational cohorts. Although there are slight international differences about the birth year of generation cohorts, we have considered the following aggregation [54]: Silent generation (people born from 1924 and 1946), Baby Boomers (1945-1964), X generation (1965-1980) and Y generation (1981-1990). This information was obtained taking into consideration the survey year and the imputed age of each individual. 
We select primary questions whether people report a low state of health (LowSelfRatedHealth). The original question was: How is your health in general? It is very good (1), good (2), fair (3), bad (4), very bad (5), don't know (8) and refusal (9). We have recodified this variable into LowSelfRatedHealth so they take the value 1 if individuals reported answers from 3 to 5, 0 otherwise. Reported answers for 8 and 9 were considered missing values.

As explanatory variables, we control for socio-demographic characteristics (age, marital status, educational level and employment) and lifestyles (smoking, overweight and lack of physical exercise).

\section{Results}

\section{Descriptive results}

Table 1 summarizes the main descriptive statistics. Older individuals report a worse self-perceived state of health than younger ones, and women worse than men. The gender gap increases with age. One potential reason is that life expectancy is higher for women, thus living longer does not mean living better. In addition, other consequence is that the percentage of married men of silent generation is much greater than the percentage of their female counterparts for whom the percentage of widows is greater. Educative levels improve among younger generation cohorts. We have to be careful with the case of $\mathrm{Y}$ generation because most of them are still enrolled in the educative system (over $40 \%$ ). For silent generation and baby boomers, educative level is higher for men than for women, whereas this situation is reversed among younger generation cohorts. There are also important gender differences for working situation along generations. The employment rate is greater for men than for women, and the other way around for the case of house-keeping. One consequence is that the percentage of pensioners is greater for men than for women of silent generation. Regarding life styles, men are more likely 
to be daily smokers than women, and the prevalence peak is reached for X generation. Men are more likely to be overweight than women, and the prevalence peak is reached for baby boomers among men and silent generation among women. Lastly, women are more sedentary than men. Gender gaps are smoothed for older cohorts.

\section{Insert here Table 1}

As expected, women value their state of health worse than men for all generation cohorts. Self-rated health gets worsen with age, finding remarkable gaps for men and women from silent generation in comparison to baby boomers and for baby boomers in comparison to men and women from $\mathrm{X}$ generation.

\section{Insert here Graph 1}

We have drawn macro data from the EUROSTAT. The Gini coefficient measures the inequality. Czech Republic, Slovakia and Slovenia are characterized by high levels of inequality (being also the most unequal inside the European Union): Latvia and Romania are the most equal (with Spain and Greece are among the most equal countries inside the European Union). To distribute countries according to the Gini index, we have taken into account Gini tertiles, thus countries included in the first group are the most unequal and countries included in the third groups are the most equal.

Insert here Table 2

Looking at national inequity levels, we observe that people living in countries in the second tertile of inequity show the best perceived health. People living in countries with the highest inequity or in the third tertile show the worse self-perceived state of health.

Insert here Graph 2

\section{Multilevel models results}

As empirical strategy, we consider multilevel models (STATA: xtmelogit). Multilevel regression models are indicated when there is a hierarchical structure in levels 
of data, with a single dependent variable measured at the lowest level and a set of explanatory variables on each of the levels. We follow the empirical strategy of the paper of Pinilla et al. (2002) [52].

In our case, our data is structured with $\mathrm{j}$-countries, in each of which $\mathrm{n}_{\mathrm{j}}$ persons have been interviewed. Our dependent variable is LowSelfRatedHealth ${ }_{i j}$ which summarizes if the individual i of country $\mathrm{j}$ reports a low state of health (1: yes; 0: otherwise). Thus, we represent this variable as:

$$
\text { LowSelfRatedHealth }_{i j}=X_{i j}^{\prime} \bar{\beta}+u_{i j}+\varepsilon_{i j}(1)
$$

in which $X$, set of explanatory variables, includes $K$ regressors. As explanatory variables, we consider socio-demographic variables (age, civil state, educational level and working status) and life-styles (smoking, overweight and sedentarism). The parameter $\bar{\beta}$ represents the fixed effects, which are dependent on $L$ national variables, $K-1$ individual variables and a constant. This model assumes that the random effects $u_{j}$ are distributed normally with mean 0 and variance $\sigma_{u}^{2}=\sigma_{\beta}^{2}$, which stands for differences in the variable LowSelfRatedHealth attributable to the country. It also assumes that the error component $\varepsilon_{i j}$ is also distributed normally with mean 0 and variance ${ }^{\sigma^{2}}$. And finally it assumes that the random effects $u_{j}$ and the error component $\varepsilon_{i j}$ are independent, and that the $\varepsilon_{i j}$ are all independent one from another.

Because our dependent variable (LowSelfRatedHealth) is binary, we applied multilevel analysis with a logistic function. The multilevel analysis helps us to understand individual and national health determinants and if national differences remain after controlling for micro variables. We repeat estimations by sub-samples of men and women. We also repeat estimation by generation cohorts. 
We also wonder if equity (Gini Tertiles) play an important role in the forming of health in general, and in the corresponding gender gap in particular. To that end, we carry out an Oaxaca analysis for LowSelfRatedHealth to address gender gaps repeating estimations by sub-samples of countries distributed in Gini Tertiles. The Blinder-Oaxaca decomposition shows if difference in the means of LowSelfRatedHealth between men and women are due to differences in the mean values of the independent variables within them and/or group differences in the effects of the independent variables.

Table 3 summarizes main results of individual characteristics and random effects.

\section{Insert here Table 3}

Age is an important predictor of the self-perceived state of health even among people who belong to the same generation. So, older men from silent generation perceive their health worse than younger men from the same generation. Male baby-boomers asse worse health self-perception with age than their female counterparts, whereas age plays a greater role for women from silent generation than for their male counterparts. Regarding civil status, it has a different impact by gender and generation cohorts. As an illustration, being widow has a stronger impact for women from silent generation than for men, whereas the impact is stronger for men from $\mathrm{X}$ and $\mathrm{Y}$ generations than for women. The impact of being widow is stronger for younger generation cohorts than for older generations.

Educational level has an important impact on the self-perceived state of health, thus higher education levels improve the self-perceived health. The impact of education is the greatest for baby-boomers. In general, health perception is more sensitive to education among women than among men. Regarding employment situation, being unemployed versus being employed exert a negative influence on the self-perceived state of health, being the impact stronger for men and older generations. Being pensioner and 
housekeeper also exert a negative influence on the self-perceived state of health. Only being student versus working has a positive influence for men from Y generation.

Smokers from younger generations perceive their health worse than non-smoker. Special case are male smokers from silent generation that consider their health better than non-daily smokers Being overweight and physically inactive is also negative for health. The negative impact of smoking and overweight on health perception is stronger for women than for men, for younger generations than for older generations. Nevertheless, the negative impact of sedentarism on health perception is, in general, stronger for men than for women, for the oldest and the youngest generations.

Random effects are relevant and statistically significant for both male and female sub-samples. Random effect models assist in controlling for unobserved heterogeneity, thus unobserved heterogeneity plays a greater role in figuring out self-perceived health. Random effects are stronger for older generations among women and for younger generations among men. In Table 4, we try to understand if it is possible to obtain an explanation of random effects based on national differences by definition of Gini tertiles.

Table 4 ratifies gender differences of Graph 2. People living in countries in the second tertile of inequity show the best perceived health for all generations except silent generation to whom higher level of inequity are associated to worse health selfperception. People living in countries with the highest inequity show the worse selfperceived state of health. Gender differences increases for older generation cohorts. The highest gender gaps are for people living in the most equal countries among silent generation and for people living in the most unequal for Baby Boomer. For people from $\mathrm{X}$ and $\mathrm{Y}$ generations, the highest gender gaps are found for people living in countries of $2^{\text {nd }}$ tertile of Gini index. Gender differences among silent generation are explained by endowment and coefficient effects for sub-samples of Gini T1 and T2, however for people 
living in most unequal countries, gender differences are not so much explained by differences on the characteristics of men and women, but by differences on the impact of these characteristics. This means, for example, that educational levels of men and women from silent generation in most unequal countries are not so different, but the impact of education on their self-perceived health. In general endowment effect plays a negative role increasing gender gaps in favor to men for older generation cohorts, but it seems that female characteristics are improving along generations thus endowment effect plays a smoother role in gender gaps in favor to women. In general coefficient effect plays a negative role increasing gender gaps in favor to men, for all generations, but specially for the silent generation.

Insert here Table 4

\section{Discussion}

Among our results we highlight that age is an important predictor of self-rated health, even among people who belong to the same generation. As in previous research, educational level is positively correlated with self-perceived state of health, whereas unhealthy lifestyles and poor working conditions are negatively correlated. This research stress out that the impact of education on health perception is stronger for women than for men. Among generation cohorts, the greatest impact is for Baby Boomers. The impact of smoking and overweight is stronger for women than for men, for younger generations than for older generations. The other way around happens for the case of sedentarism. As the European Commission (2013) [53] reports these facts reflect the influence that stressful life conditions have on both health and health-related behaviors among vulnerable population groups. The differences found in youth are likely to be maintained in adulthood and involve the persistence of sexist attitudes regarding the expression of discomfort, differential treatment of ailments and work and domestic work [25]. The 
educational and health system should take the corresponding preventive measures aimed at a model that promotes gender equality.

As main research implication, we conclude that it is important to take into account differences based on gender, generation cohorts and national settings. It is relevant that people who worse value their health are people living in the most unequal and the most equal countries. Regarding healthy aging, people from the silent generation show the highest gender gaps in relation to self-perceived health. In this case characteristics and their impact on health explain gender differences for equal countries, but in case of the most unequal countries, gender differences on characteristics (endowment effects) are not so important as gender differences on characteristics' impact (coefficient effects) [53].

The scientific analysis of these health inequalities at national level is a complex topic because we need to isolate the effect of several indicators interrelated. In this paper we have selected Eastern European countries because they share a common welfare system, and there is an important linkage among equity and capacity to create richness. In spite of the results, inequity could be studied as mediating variables that also affect to structural and cultural changes [27]. How income inequality harms population health is still an open debate [43]. In fact, this analysis has to do with broader debates about progress and sustainability $[27,54]$.

Although self-reported health may not always accurately capture variations in absolute health across countries, the ease, speed and economy of collecting self-reports of health (even with a single item global question such as the one used here) make such collection attractive for rapid appraisals. Also, collecting self-reports of health will make it easier to empirically assess epidemiologic associations between various exposures and health, especially in countries where objective health data are lacking [55]. 
For future research, limitations derived from the use of self-perceived health might be handled by including objective health indicators like limitations in daily activities. Another research line will be to analyze the impact of domestic health systems in the perception of health. In this sense, differences in coverage and access may be relevant in the assessment of citizens' health. A longitudinal approach of these questions, for example by a panel data strategy based on individuals, regions and nations would provide information on the impact of the economic crisis and its possible recovery according to wealth indicators.

\title{
Conclusions
}

Despite the important role of unobserved heterogeneity figuring out self-perceived health, especially for men and older generation, our results show that in Eastern Europe persists a gender gap in self-assessed health, with relevant differences on health determinants by generation cohorts, even when controlling for countries with different contexts. Historical, sociocultural, political and economic context is therefore critical to understand health trajectories of women and men, which are not uniform across cohorts [21]. In order to narrow the self-rated health gender gap, policy strategies have to take into consideration the cohort weight in sharing different living conditions and social representations, attitudes and values which integrates this perception.

\author{
Abbreviations \\ EUROSTAT: Statistical Office of the European Union \\ GDPpc: Gross Domestic Product per capita \\ EHIS: European Health Interview Survey
}




\section{Declarations}

Ethics approval and consent to participate: We used data drawn from the European Health Interview Survey for 8 Eastern European Countries from 2006 through 2009 (Eurostat). Eurostat grants access to microdata only to recognised research entities. To qualify for recognition, an organisation must: • have research as one of its main activities (e.g. universities, research institutions) or be a research department within other organisation (e.g. within bank, statistical institute, etc.); • provide evidence of publication of research results; - be independent and autonomous in formulating scientific conclusions; • have adequate data security safeguards. Responsibilities Before being recognized as a research entity, an organisation must sign a confidentiality undertaking with the terms of use. The confidentiality undertaking commits the signatory and all researchers having access to confidential data to: • accessing confidential data only for the agreed purposes and $\bullet$ guaranteeing the physical security of the data, including prevention and taking action in case of violation of confidentiality.

Consent for publication: Not applicable.

Availability of data and materials: Database by waves is available on Internet:

European Health Interview Survey Web Page:

https://ec.europa.eu/eurostat/web/microdata/european-health-interview-survey

Competing interests: The authors declare that they have no competing interests.

Funding: This research was funded by the Government of Aragón (Reference Group BYCS S16_17R) and co-financed with Feder 2014-2020 “Building Europe from Aragón" and Ministry of Economy, Industry and Competitiveness, Government of Spain, grant number: "CSO2017-82110-R". These funders had no role in the design of 
the study, in the collection, analysis, and interpretation of the data, or in the writing or approval of the manuscript.

Authors' contributions MGL and AIGL conceived and designed the study, AIGL supervised the creation of the study dataset, planned and conducted the statistical analyses, MGL, AIGL and IAP wrote the paper. MGL, AIGL and IAP reviewed the results and provided input on the manuscript. All authors read and approved the final manuscript.

Acknowledgements: The authors would like to acknowledge European citizen who answered this survey and made possible this research.

\section{References}

1. Mäki N, Martikainen P, Eikemo T, Menvielle G, Lundberg O, Östergrene O, et al. Educational differences in disability-free life expectancy: a comparative study of long-standing activity limitation in eight European countries. Soc Sci Med. 2013; 94: $1-8$.

2. Bird CL, Rieker PP. Gender matters: an integrated model for understanding men's and women's health. Soc Sci Med. 1999; 48: 745-55.

3. Jakab, Z. Foreword. In WHO. Women's health and well-being in Europe: beyond the mortality advantage. Copenhagen: WHO Regional Office For Europe, 2016-

4. Karanikolos M, Leon DA, Smith PC, McKee M. Minding the gap: changes in life expectancy in the Baltic States compared with Finland. J Epidemiol Community Health. 2012; 66: 1043-9. 
5. Cambois E, Solé-Auró A, Brønnum-Hansen H, Egidi V, Jagger C, Jeune B et al. Educational differentials in disability vary across and within welfare regimes: a comparison of 26 European countries in 2009. J Epidemiol Community Health. 2016; 70: $331-8$.

6. Marmot M, Allen J, Bell R, Bloomer E, Goldblatt P. WHO European review of social determinants of health and the health divide. Lancet. 2012; 380 (9846): 1011-29.

7. Dahlin J, Haärkönen J. Cross-national differences in the gender gap in subjective health in Europe: Dose country level gender equality matter? Soc Sci Med. 2013; 98: 24-8.

8. Girón P. Los determinantes de la salud percibida en España. Madrid: Universidad Complutense de Madrid; 2010.

9. Idler EL, Benyamini Y. Self-rated health and mortality: a review of twenty-seven community studies. J Health Soc Behav. 1997; 38: 21-37.

10. Souto S. Percepción de la salud, determinantes sociales en la atención primaria. P.h.D. Thesis, Coruña: Universidad de A Coruña; 2012.

11. Zúñiga MA, Fos JGT, Carrillo PJ, Gandek B, Medina MMR. Evaluación del estado de salud con la encuesta SF-36: resultados preliminares en México. Salud Publica Mex. 1999; 41 (2): 110-8

12. De Salvo K, Fan VS, McDonell MB, Fihn SD. Predicting mortality and healthcare utilization with a single question. Health Serv Res. 2005; 40: 1234-46.

13. Devaux M. Income-related inequalities and inequities in health care services utilisation in 18 selected Organisation for Economic Cooperation and Development countries. Eur J Health Econ. 2015; 16: 21-33.

14. Zimmer Z, Natividad J, Lin HS, Chayovan NA. cross-national examination of the determinants of self-assessed health. J. Health Soc. Behav. 2000; 41:465-81. 
15. Gil-Lacruz M. Psicología social aplicada al bienestar. Zaragoza: Prensas Universitarias; 2007.

16. Schwartzmann L. Calidad de vida relacionada con la salud, aspectos conceptuales. Cienc enferm. 2003; 9 (2): 9-21.

17. Friedman H. Health Psychology Handbook. Oxford: Oxford Books; 2011.

18. Tuesca-Molina R, Centeno RH, Ossa SM, García DN, Lobo LJ. Calidad de vida relacionada con la salud y determinantes sociodemográficos en adolescentes de Barranquilla (Colombia). Salud Uninorte. 2008; 24 (1): 53-63.

19. Sperlich S, Tetzlaff J, Geyer S. Trends in good self-rated health in Germany between 1995 and 2014: do age and gender matter? Int J Public Health 2019; 64: 921.

20. Debiasi E. Age-period-cohort analysis: a summary of analytical approaches and results. Centre for Economic Demography and Department of Economic History, Lund University; 2018.

21. Etherington N. Re-evaluating gender differences in self-rated health: The importance of cohort. J. Women Aging 2017; 29 (2): 150-62.

22. Crossley T, Kennedy S. The reliability of self-assessed health status. J Health Econ. $2002 ; 21: 643-58$.

23. Schnittker J. Education and the Changing Shape of the Income Gradient in Health. J Health Soc Behav. 2004; 45: 286-305.

24. Wang J, Smith W, Cumming R, Mitchell P. Variables Determining Perceived Global Health Ranks: Findings from a Population_based Study. Ann Acad Med. 2006; 35: $190-7$.

25. Gil-Lacruz M, Gil-Lacruz AI. Calidad de vida relacionada con la salud, ¿cómo influye el nivel educativo? Proceeding of the I International Congress, Health, 
Education and Quality of Live; 2016. Lérida: GESEC Societat, Salut, Educació i Cultura.

26. World Health Organisation Commission (WHO) on Social Determinants of Health. Closing the Gap in a Generation: Health Equity through Action on the Social Determinants of Health. Final Report. Geneva: WHO; 2008.

27. Ekersley R. Beyond inequality: Acknowledging the complexity of social determinants of health. Soc Sci Med. 2015; 147: 121-5.

28. Denton M, Prus S, Walters V (2004) Gender differences in health: a Canadian study of the psychosocial, structural and behavioural determinants of health. Soc Sci Med. 2004; 58:2585-600.

29. Hill TD, Needham BL. Gender-specific trends in educational attainment and selfrated health, 1972-2002. Am J Public Health.2006; 96:1288-92

30. Aguilar-Palacio I, Carrera-Lasfuentes P, Sánchez-Recio R, Alonso JP, Rabanaque MJ. Recession, employment and self-rated health: a study on the gender gap. Public Health 2018; 154:44-50

31. Assari S, Preiser B, Kelly M. Education and Income Predict Future Emotional WellBeing of Whites but not Blacks: A Ten-Year Cohort. Brain Sci. 2018, 8(7): 122.

32. Sen A. Health: perception versus observation. BMJ. 2002; 324: 860-1.

33. Adams SA, Matthews CE, Ebbeling CB, Moore CG, Cunningham JE, Fulton J, et al. The effect of social desirability and social approval on self-reports of physical activity. Am J Epidemiol. 2005; 161(4): 389-98.

34. Ekersley R. Well \& Good: Morality, Meaning and Happiness. Melbourne: Text Publishing; 2005. 
35. Biggs B, King L, Basu S, Suckler D. Is wealthier always healthier? The impact of national income level, inequality and poverty on public health in Latin America. Soc Sci Med. 2010; 71: 266-73.

36. Van de Poel E, Van Doorslaer E, O’Donnell O. Measurement of inequity in health care with heterogeneous response of use to need. J Health Econ. 2012; 31: 676-89.

37. Jylhä M, Guralnik JM, Ferruci L, Jokela J, Heikkinen E. Is self-rated health comparable across cultures and genders? J Gerontol Series B, Psychological Sciences and Social Sciences. 1998; 53 (3): 144-52.

38. Zagorski K, Evans MDR, Kelley J, Piotrowska K. Does national income inequality affect individuals quality of life in Europe? Inequality, Happiness, Finances and Health. Soc Indic Res. 2014; 117: 1089-110.

39. Huijts T, Monden CWS, Kraaykamp G. Education, education hierogamy, and selfassessed health in Europe: a multilevel study of spousal effects in 29 countries. Eur Sociol Rev. 2010; 26 (3): 261-76.

40. Deaton A. Health, inequality, and economic development. J Econ Lit. 2003; 41 (1): $113-58$.

41. Dollar D, Kray A. Growth is a good for the poor. Econ J. 2002; 7 (3): 195-225.

42. Wilkinson RC, Pickett KE. Income inequality and population health: a review and explanation of evidence. Soc Sci Med. 2006; 62(7): 1768-84.

43. Hu Y, Van Lenthe FJ, Mackebach JP. Income inequality, life expectancy and causespecific mortality in European countries, 1987-2008: a fixed effects study. Eur J Epidemiol. 2015; 30: 615-25.

44. Calling S. Longitudinal trends in good self-rated health: effects of age and birth cohort in a 25-year follow-up study in Sweden. Int J Public Health. 2015; 60 (3): 363-73. 
45. Sacker A, Worts D, McDonough P. Social influences on trajectories of self-rated health: evidence from Britain, Germany, Denmark and the USA. J Epidemiol Community Health.2011; 65(2): 130-6.

46. Zack MM, Moriarty DG, Stroup DF, Ford ES, Mokdad AH. Worsening trends in adult health-related quality of life and self-rated health United States, 1993-2001. Public Health Rep. 2004; 119 (5): 493-505.

47. Badley EM, Canizares M, Perruccio AV, Hogg-Johnson S, Gignac MA. Benefits Gained, Benefits Lost: Comparing Baby Boomers to Other Generations in a Longitudinal Cohort Study of Self-Rated Health. Milbank Q. 2015; 93: 40-72.

48. Chen H, Cohen P, Kasen S. Cohort differences in self-rated health: evidence from a three-decade, community-based, longitudinal study of women. Am J Epidemiol. 2007; 166(4): 439-46.

49. Salonna F, Geckova AM, Zezula I, Sleskova M, Grothoff JW, Reijneveld SA, et al. Does social support mediate or moderate socioeconomic differences in self-rated health among adolescents? Int J Public Health Res. 2012; 57(3): 609-17.

50. Braveman P, Egerter S, Williams D. The Social Determinants of Health: Coming of Age. Annu Rev Public Health. 2011; 32: 381-98.

51. EUROSTAT Questionaire of the European Health Interview Survey. 2016 [Cited 2017 Jan 12] Available from http://ec.europa.eu/eurostat/web/microdata/europeanhealth-interview-survey.

52. Pinilla J, González B, Barber P, Santana Y. Smoking in young adolescents: an approach with multilevel discrete choice models. J Epidemiol Community Health. 2002; 56: 227-32. 
53. European Commision. Health inequalities in the EU. Final Report of the Consortium. Consortium leader: Sir Michael Marmot. Geneva: European Commision Directorate General for Health and Consumers, 2013.

54. Costanza R, Kubiszewski I, Giovannini E, Lovins H, McGlade J, Pickett KE et al. Time to leave GDP behind. Nature. 2014; 505: 283-5.

55. Subramanian SV, Huijts T, Avendano M. Self-reported health assessments in the 2002 World Health Survey: how do they correlate with education? Bull World Health Organ. 2010; 88: 131-8.

\section{FIGURE TITLES / LEGENDS}

Graph 1. Prevalence of low self-rated health among men and women. Data in percentages Source: Self-elaborated graph with data drawn from European Health Interview Survey and Eurostat.

Graph 2. Prevalence of low self-rated health among men and women of different generations by GINI tertiles. Data in percentages Source: Self-elaborated graph with data drawn from European Health Interview Survey and Eurostat. 